\title{
Role of C- reactive protein in diabetic retinopathy
}

\author{
May $D^{1}$, Francis EA $R^{2}$, Norman $M^{3}$, Nelly $N^{4}$, Nameeth $D^{5}$, Shah A. $J^{6}$ \\ ${ }^{1}$ Dr Dsouza May, ${ }^{2}$ Dr Rodrigues Francis EA, ${ }^{3}$ Dr Mendonca Norman, ${ }^{4}$ Dr Nazareth Nelly DO, DNB, above all \\ authors are affiliated with Father Muller Medical College, Mangalore, ${ }^{5}$ Dr Dsouza Nameeth, FVRS, KS Hegde \\ Medical Academy, Mangalore, ${ }^{6}$ Dr Shah Amish J, Aravind Eye Hospital, Madurai, India.
}

Address for Correspondence: Dr May D’Souza, 3-30-2476, D’Souza Compound C/o Pharma Promoters, Kadri Kambala Junctiom, Mangalore, Karnataka. Email: maydsouza22@gmail.com

\begin{abstract}
Introduction: Diabetes mellitus is a major medical problem throughout the world. Diabetes causes an array of long-term systemic complications that have considerable impact on the patient as well as society, as the disease typically affects individuals in their most productive years. C Reactive Protein is an acute phase reactant and an inflammatory mediator involved in the pathogenesis of Diabetic Retinopathy. Aim: The aim of this study is to determine the correlation of C-reactive protein with diabetic retinopathy and different grades of retinopathy. Methods: The study was done on 200 diabetic patients attending the ophthalmology outpatient department, who were selected by purposive sampling. The patients were subjected to a complete ocular examination. Based on the fundus examination patients were categorized into the respective study groups: - 1) Controls 2) Diabetics without Diabetic Retinopathy 3) NPDR 4) PDR 5) CSME. The patients were then subjected to C- reactive protein estimation via laboratory analysis (Turbidometry Technique). Results: The study showed that the mean CRP levels in diabetics were found to be $3 \mathrm{mg} / \mathrm{dl}$ which is well within normal limits. The CRP levels were relatively higher in the PDR and CSME groups with it being elevated in $30 \%$ and $20 \%$ patients in the groups respectively. Conclusion: CRP levels in our study did not show significant correlation with diabetes mellitus. Although higher grades of retinopathy had higher CRP levels, the correlation was not significant and consistent. Hence C- reactive protein cannot be used as a reliable screening tool for diabetic retinopathy.
\end{abstract}

Key words- Diabetic retinopathy, C- reactive protein, Pathogenesis, Diabetes mellitus, PDR, CSME

\section{Introduction}

There are approximately 35 million people in India with diabetes, the largest number in any country around the world and this is predicted to rise to 80 million by 2030. Diabetes is known to cause microangiopathy and affects small caliber vessels leading to end organ damage like Diabetic Retinopathy (DR). Although progress from no symptoms to blindness can take several decades in diabetic retinopathy, it already accounts for $3 \%$ blindness in India.

According to the Chennai Urban Rural Epidemiology Eye Study (CURES) the prevalence of diabetic retinopathy in South India is $17.6 \%$ and this is a large number [1]. There is no national

Manuscript received: $6^{\text {th }}$ February 2017 Reviewed: $16^{\text {th }}$ February 2017

Author Corrected: $24^{\text {th }}$ February 2017

Accepted for Publication: $2^{\text {nd }}$ March 2017 screening program in India at the moment for diabetic retinopathy because it is difficult to plan and monitor and would be expensive. The need for a simple, inexpensive, repeatable and fairly reliable screening test is the need for the present times for the timely detection of diabetic retinopathy, such that if required, patients can be redirected to higher centers for further management in order to prevent any untoward complications. In order to prevent blindness from diabetic retinopathy, prevention and early detection are mandatory.

Thus education of the masses about the prevalence and risk factors of diabetic retinopathy, identifying high risk groups among the diabetics, adequate glycaemic control and using cost effective screening methods in order to aid in diagnosis and timely intervention are the prerequisites for the 
control of this epidemic of diabetic retinopathy. Inflammation is now recognized as a critical contributor in the pathogenesis of diabetic retinopathy by contributing to the atherothrombotic process.

Studies have shown the role of coagulation factors in diabetic retinopathy in both type $1 \&$ type 2 diabetes mellitus. In addition, various inflammatory markers have been implicated in the disease process and progression of diabetic retinopathy.

C-reactive protein was discovered in 1930 , but it was some time later that it was found that the Creactive protein level in a person's blood is an important and highly accurate predictor of future heart disease. C-reactive protein (CRP) is a sign of inflammation in the walls of arteries.

The studies showed that reducing inflammation by lowering CRP levels with a class of drugs known as statins significantly lowered the rate of heart attacks.

C- reactive protein is one such well known inflammatory marker and acute phase reactant. Its relative diagnostic and prognostic role have determined in many musculoskeletal disorders, liver and kidney disorders. But few attempts have been made to show its role in diabetic patients especially in those with retinopathic changes.

Cheung in his study showed that retinal microvascular caliber is associated with higher levels of highly sensitive CRP (hs-CRP) in Asians [2]. This supported the concept that retinal venular calibre may be a marker for low-grade systemic inflammation.

Kilpatrick et al., in their study on CRP concentrations in Type I diabetics found it to be raised yet within the normal range [3]. This study found that factors already known to be associated with cardiovascular risk in type I diabetes (duration of diabetes, current smoking status, lipid status, hypertension and microvascular complications) are also indicative of raised CRP concentrations.

In this study an attempt was thus made to see if $\mathrm{C}$ reactive protein can prove to be a marker for the detection of diabetic retinopathic changes in diabetic patients.

\section{Aims and Objectives}

To determine the correlation of $\mathrm{C}$ - reactive protein with

- Diabetic retinopathy

- Different grades of retinopathy

\section{Materials and Methods}

It was an analytical study done on patients attending the ophthalmology outpatient department at Father Muller Medical College \& Hospital from January 2011 to June 2012. All study procedures adhered to the principles outlined in the declaration of Helinski for research involving human subjects and clearance was obtained from the institutions ethics committee. Informed consent was obtained from all willing participants. 200 patients satisfying the inclusion and exclusion criteria given below were selected by purposive sampling.

\section{Selection Criteria}

\section{Inclusion Criteria}

- Established cases of diabetes mellitus who were on oral hypoglycemic agents or insulin for at least $1 \mathrm{yr}$ from date of diagnosis

- All study subjects should be having normal hemogram, liver function tests \& kidney function tests

- $\quad$ Both Type I \& Type II DM

\section{Exclusion Criteria}

- Patients suffering from any systemic infective conditions - liver \& kidney diseases, rheumatoid arthritis, tuberculosis, carcinoma, pyrexia of unknown origin.

- Patients with coexistent infective or inflammatory ocular conditions- stye, conjunctivitis, uveitis, scleritis etc.

- Pregnant women.

- Patients who underwent previous photocoagulation therapy

- In case of controls - No family h/o diabetes mellitus (in addition to other exclusion criteria)

A detailed history was obtained from each patient followed by a complete ocular examination including determination of visual acuity and detailed fundus examination using the slit lamp \& indirect ophthalmoscope. 
After the fundus examination patients were categorized based on the Early treatment Diabetic Retinopathy Study (ETDRS) [4] grading into the respective study groups of :-

Group 1 - Control

Group 2 - DM without Diabetic retinopathy

Group 3 - Non Proliferative Diabetic retinopathy

Group 4 - Proliferative Diabetic retinopathy

Group 5 - Clinically significant Macular Edema
The patient was then subjected to $\mathrm{C}$ - reactive protein estimation via laboratory analysis (Turbidometry Technique)

Fundus Examination- For a detailed fundus examination the pupils were dilated with tropicamide $0.8 \%$ and phenylephrine drops 5\% (1 drop every ten minutes for one hour duration), following which patient was subjected to indirect ophthalmoscopy and slit lamp examination with a 90D lens.

Statistical Analysis- Data was analyzed by the Chi Square Test and Analysis Of Variance (ANOVA).

\section{Results}

In this study patients satisfying the inclusion and exclusion criteria were selected and divided into 5 study groups which were the following:

1. Controls

2. Diabetics with no Diabetic Retinopathy

3. Non Proliferative Diabetic Retinopathy (NPDR)

4. Proliferative Diabetic Retinopathy(PDR)

5. Clinically Significant Macular Edema (CSME)

40 patients each were taken, in each of these study groups.

Age Distribution- In the study, the youngest patient was 23 years old and oldest 88 years. Of the total patients the mean age group in this study was around 60 years. The mean age, individually in the various study groups ranged between 40-60 years which constitutes the middle age group.

Diabetic Duration- Of the diabetics who were included in the study majority of the patients were diagnosed with diabetes since approximately 10 years. The minimum years since diagnosis of diabetes was made in the patients, was 1 year and maximum was 30 years.

Sex Distribution- 40 patients each were taken of the above categories. In all the groups the ratio of males is higher compared to females, except in the CSME group where the male to female ratio is equal.

Of the total 200 patients in the study, 118 are male and 82 are female thereby showing an overall male preponderance in the incidence of diabetes in the study population.

Grades of NPDR- In the NPDR study group majority of the patients (50\%) suffered from moderate degree of retinopathy. Least belonged to the very severe NPDR category (10\%).

Blood Sugar Levels- The mean Random Blood Sugar (RBS) ranged from 200-250 mg/dl in the Diabetic patients. The PDR and CSME study groups had a higher mean blood sugar ranging between $300-350 \mathrm{mg} / \mathrm{dl}$ indicating the correlation between higher severe sugar and the severe grades of retinopathy.

HbA1C Levels- In the above graph it is clearly evident that the HbA1C levels are elevated in the patients with NPDR, PDR and CSME. A significant correlation especially was found with HbA1C in patients with PDR and CSME (p-0.000) 
Fundus Examination- Majority of the patients with diabetes have no history of previous fundus examination. For $67.5 \%$ of the patients this was the first fundus examination they underwent. Of these patients, 28 patients were newly diagnosed with NPDR and 24 patients each belonging to the CSME and PDR groups were newly diagnosed. [Table 1]

Table-1: History of previous fundus examination in the diabetic study groups.

\begin{tabular}{|c|c|c|c|c|c|c|}
\hline \multirow{2}{*}{} & \multicolumn{5}{|c|}{ Group } & \\
\cline { 3 - 7 } & & DM without DR & NPDR & PDR & CSME & Total \\
\hline PREV & N & 32 & 28 & 24 & 24 & 108 \\
\hline FUNDUS & EXMN & $80 \%$ & $70 \%$ & $60 \%$ & $60 \%$ & $67.5 \%$ \\
\hline & Y & 8 & 12 & 16 & 16 & 52 \\
\hline & & $20 \%$ & $30 \%$ & $40 \%$ & $40 \%$ & $32.5 \%$ \\
\hline & Total & 40 & 40 & 40 & 40 & 160 \\
\hline & & $100 \%$ & $100 \%$ & $100 \%$ & $100 \%$ & $100 \%$ \\
\hline
\end{tabular}

CRP Levels- The mean CRP levels in all the patients was found to be $3 \mathrm{mg} / \mathrm{L}$. In all the grades of diabetic retinopathy the mean value of CRP was found to be less than $5 \mathrm{mg} / \mathrm{L}$, therefore within normal limits.

Thus CRP correlation with diabetic retinopathy is not significant as no considerable elevation was noticed [Table 2].

\section{Table 2: CRP Levels in the Study Groups}

The CRP levels in all the controls and diabetics without DR was within normal limits. In majority patients CRP levels in the PDR and CSME groups were again within normal limits.

Only 20-30\% patients in the PDR and CSME group had elevated CRP levels the highest being 6.5mg/L.

Table 2: History of previous fundus examination in the diabetic study groups.

\begin{tabular}{|c|c|c|c|c|}
\hline & & \multicolumn{2}{|c|}{ CRP } & \multirow[b]{2}{*}{ Total } \\
\hline & & Normal & Abnormal & \\
\hline \multirow[t]{10}{*}{ Group } & Control & 40 & 0 & 40 \\
\hline & & $100.0 \%$ & $0 \%$ & $100.0 \%$ \\
\hline & DM without DR & 40 & 0 & 40 \\
\hline & & $100.0 \%$ & $0 \%$ & $100.0 \%$ \\
\hline & NPDR & 38 & 2 & 40 \\
\hline & & $95.0 \%$ & $5.0 \%$ & $100.0 \%$ \\
\hline & PDR & 28 & 12 & 40 \\
\hline & & $70.0 \%$ & $30.0 \%$ & $100.0 \%$ \\
\hline & CSME & 32 & 8 & 40 \\
\hline & & $80.0 \%$ & $20.0 \%$ & $100.0 \%$ \\
\hline \multirow{2}{*}{\multicolumn{2}{|c|}{ Total }} & 178 & 22 & 200 \\
\hline & & $89.0 \%$ & $11.0 \%$ & $100.0 \%$ \\
\hline
\end{tabular}

Fishers exact test $p=004$, HS 
Table-3: Correlation of the various variables in the study groups.

\begin{tabular}{|c|c|c|c|}
\hline & & $\begin{array}{c}\text { Karl Pearson } \\
\text { Correlation r value }\end{array}$ & p Value \\
\hline \multirow[t]{5}{*}{ AGE } & YRS SINCE DIAGNOSED & $.510 * *$ & .000 \\
\hline & CRP & $.338 * *$ & .001 \\
\hline & RBS & .118 & .285 \\
\hline & FBS & $.423 * *$ & .000 \\
\hline & HBA1C & $.249 *$ & .047 \\
\hline \multirow[t]{5}{*}{ YRS SINCE DIAGNOSED } & AGE & $.510 * *$ & .000 \\
\hline & CRP & $.508 * *$ & .000 \\
\hline & RBS & $.344 * *$ & $.344 * *$ \\
\hline & FBS & $.466 * *$ & .000 \\
\hline & $\mathrm{HbA} 1 \mathrm{C}$ & $.350 * *$ & .005 \\
\hline \multirow[t]{5}{*}{ CRP } & AGE YRS SINCE & $.338 * *$ & .001 \\
\hline & DIAGNOSED & $.506 * *$ & .000 \\
\hline & RBS & $.527 * *$ & .000 \\
\hline & FBS & $.590 * *$ & .000 \\
\hline & $\mathrm{HbA} 1 \mathrm{C}$ & $.350 * *$ & .005 \\
\hline \multirow[t]{5}{*}{ RBS } & AGE YRS SINCE & .118 & .285 \\
\hline & DIAGNOSED & $.344 * *$ & .004 \\
\hline & CRP & $.527 * *$ & .000 \\
\hline & FBS & $.934 * *$ & .000 \\
\hline & $\mathrm{HbA} 1 \mathrm{C}$ & $.726 * *$ & .000 \\
\hline \multirow[t]{5}{*}{ FBS } & AGE YRS SINCE & $.423 * *$ & .000 \\
\hline & DIAGNOSED & $.466^{* *}$ & .000 \\
\hline & CRP & $.590 * *$ & .000 \\
\hline & RBS & $.934 * *$ & .000 \\
\hline & $\mathrm{HbA} 1 \mathrm{C}$ & $.713 * *$ & .000 \\
\hline \multirow[t]{5}{*}{ HbA1C } & AGE YRS SINCE & $.249 *$ & .047 \\
\hline & DIAGNOSED & $.350 * *$ & .005 \\
\hline & CRP & $.350 * *$ & .005 \\
\hline & RBS & $.726 * *$ & .000 \\
\hline & FBS & $.713 * *$ & .000 \\
\hline
\end{tabular}

** Correlation is significant at the 0.01 level ( 2 -tailed )

* Correlation is significant at the 0.05 level (2 tailed )

Correlation of various variables to one another was also noted [Table 3].

\section{Discussion}

India is becoming one of the diabetic capitals in the world. With this ever growing diabetic population, the complications due to diabetes are also growing. Diabetes is a known cause of microvascular angiopathy which leads to end organ damage, one of the organs is the eye leading on to diabetic retinopathy. The lack of education and access to medical facilities in India make the diagnosis and timely treatment of diabetic retinopathy a huge hurdle. Due to neglect, most patients end up with severe disabling or blinding disease, for which often, nothing much can be done and the patient ends up with blindness. The onset of diabetic retinopathy varies and depends on a number of predisposing risk factors, the main ones being glycaemic control and duration of onset of diabetes. Due to the unpredictable nature of blood sugar levels, a diabetic patient needs to have a regular blood check up and physician review for timely control to prevent complications. In a similar way, the role of a periodic eye check up in diabetic patients can't be overstressed as the onset and progress of diabetic retinopathic changes is not predictable and cannot be determined. 
In this study an attempt was made to asceratain the role of C- reactive protein in Diabetic Retinopathy and its correlation with different grades of retinopathy. The pathogenesis of diabetic retinopathy involves many inflammatory mediators which thus gives us an array of tests to detect these mediators. In this study we try to investigate the role of one such mediator, the $\mathrm{C}$ - reactive protein. This could serve as a initial screening tool for diabetic retinopathy when the patient undergoes his routine blood check up. This study thus aimed at finding a correlation between CRP and diabetic retinopathy and different grades of retinopathy

This study was an analytical study, spanning over eighteen months and included a total of 200 patients. Using purposive sampling the patients were segregated into study groups. Of the patients, 40 were belonging to the control group and were non diabetics with no family history of diabetes.

The remaining 160 patients belonging to the study group were diagnosed cases of diabetes, who were on treatment for diabetes with oral hypoglycaemics or insulin for at least for one year.

40 patients each belonging to the following groups,

\section{- Diabetics without DR \\ - $\quad$ Non Proliferative DR \\ - $\quad$ Proliferative DR \\ - $\quad$ CSME}

were taken who satisfied the inclusion and exclusion criteria and had no other systemic conditions (which could bring about a raised CRP), were taken as subjects in each of the study groups.

They underwent a complete ophthalmic examination including visual acuity assessment, IOP measurement, anterior segment examination and a thorough posterior segment examination with slit lamp and indirect ophthalmoscopy. They were then classified into the various study groups based on the ETDRS criteria. Then the patient was subjected to C- Reacitve Protein Analysis along with their routine blood investigations.

C -Reactive protein was estimated in the laboratory using immunoturbidometric assay. The test principle is based on the fact that Human CRP agglutinates with latex particles coated with monoclonal anti- CRP antibodies. The aggregates are then determined turbidmetrically. In normal healthy individuals CRP is a trace protein with a range up to $5 \mathrm{mg} / \mathrm{L}$. Anything above this level is considered raised.

In this study of the 160 diabetic patients, three patients were type I diabetics and 77 were type II diabetics. The average duration of diabetes in the patients was 10 years and the average blood sugar in patients was $300-350 \mathrm{mg} / \mathrm{dl}$. This again signifies the fact that poor glycaemic control predisposes to diabetic retinopathy. Nearly $60 \%$ of the patients were males indicating a higher incidence of DR in them or just the fact that the level of health education and awareness among women is poorer.

The HbA1C levels were significantly higher in those with higher grades of retinopathy and the $\mathrm{p}$ value being $<0.00$ indicated that the correlation is highly significant. This again stresses on the fact that long term glycaemic control (over 3 months), which HbA1C levels are an indicator of when elevated are associated with faster progression of diabetic retinopathy.

In this study among the diabetic patients, $67.5 \%$ although were known cases of diabetes for more than a year gave no previous history of fundus examination. Thus most of the patients were newly detected cases of diabetic retinopathy during the ophthalmic evaluation despite being diabetics from many years. This signifies the ignorance among people about the disease and its consequences and the lack of eye education with regards to diabetic retinopathy. Importance of a yearly fundus examination needs to be stressed to the general public and a general physician could also play a huge role in this as the patient regularly follows up for sugar control. Elevations in the level of markers of inflammation attributed to hyperglycemia, advanced glycation end-products, and increased BMI have been found in persons with type 1 diabetes [5,6].

While inflammatory biomarkers such as serum fibrinogen, tumor necrosis factor alpha, and Creactive protein may be elevated, there are few data regarding the role of inflammation in the progression of diabetic retinopathy. In two casecontrol studies, diabetic subjects with macular edema or PDR had higher levels of vascular endothelial growth factors and cytokines in their 
vitreous than those without macular edema or PDR [7]. In a cross-sectional study of normotensive persons with type 1 diabetes, CRP and fibrinogen levels were positively associated with diabetic retinopathy severity. In a case series of 93 diabetic patients, serum chemokines were significantly elevated in patients with at least severe nonproliferative diabetic retinopathy compared with those who had less severe retinopathy [8].

Data from the Hoorn study in persons with type 2 diabetes also showed retinopathy to be associated with serum CRP and Soluble Intercellular Adhesion Molecule-1 (sICAM-1) levels, a marker of endothelial dysfunction.

While some treatments for chronic macular edema have been focused on use of intravitreal antiinflammatory agents e.g., steroids, there are few data showing that decreasing inflammatory activity stops the development or progression of diabetic retinopathy or restores visual acuity.

Dornan et al., in their study measured plasma coagulation factors in patients of three groups; non diabetics, diabetics without retinopathy and diabetics with background and proliferative retinopathy [9]. The plasma concentrations of coagulation factors did not correlate with creatinine clearance and there were no significant differences between the groups in concentrations of C-reactive protein suggesting that the raised concentrations of coagulation factors in diabetics with retinopathy were not a result of associated nephropathy or an 'acute phase protein' response to diabetic tissue damage. Increased coagulation activity in diabetics may thereby contribute to the development of retinopathy. Schalwjk et al in their study showed that plasma concentrations of C-reactive protein were higher in Type I diabetic patients without (clinical) macroangiopathy than in control subjects, probably due to a chronic hepatic inflammatory response [10].

The correlation of C-reactive protein with markers of endothelial dysfunction suggested a relation between activation of the endothelium and chronic inflammation. In our study, no significant correlation was found between CRP and Diabetic Retinopathy. The mean value of CRP was around $3.6 \mathrm{mg} / \mathrm{L}$ among the diabetic subjects, which fell well within the normal limits.
This shows that CRP as an inflammatory mediatiors is not raised in general in diabetic retinopathy and does not have a significance in diabetic patients without any systemic involvement.

It was seen that higher grades of retinopathy (PDR and CSME) showed relatively higher values of CRP and was raised in $30 \%$ and $20 \%$ of patients respectively. Thus the sensitivity of the test in PDR and CSME is $30 \%$ and $20 \%$ respectively.

These values being low therefore make the test not reliable as a screening tool for even higher grades of diabetic retinopathy as the correlation even though present is not consistent enough to make a diagnosis of the probability of PDR and CSME being there.

Limitations of The Study- Some of the limitations of this study were the fact that Diabetes being a systemic disease tends to involve multiple systems especially renal system. They are prone to other opportunistic infections and serve as a reservoir for multiple sources of infection and therefore inflammation. Thus to get patients satisfying the inclusion and exclusion criteria of excluding other sources of infections posed a difficult task especially in those with higher grades of retinopathy.

The use of hs CRP in which a tracer elevation could have provided better insight, which at our setup was unavailable would have proven a more accurate analysis. Factors like blood sugar level, diabetic duration, prolonged glycemic control could act like confounding variables for the levels of CRP and may play a role in its blood concentration at the time of testing, as it an acute phase reactant.

Multiple CRP readings in each of the patients at different intervals would have possibly given a wider and better picture as far as the correlation which was not possible due to financial constraints.

\section{Conclusion}

The current study shows that $\mathrm{C}$ - reactive protein levels do not show significant correlation with Diabetes Mellitus. Although higher grades of retinopathy had higher CRP levels, the correlation was not significant. Hence $\mathrm{C}$ - reactive protein cannot be used as a reliable screening tool for Diabetic Retinopathy. 
List of Abbreviations

CRP - C Reactive Protein

DM - Diabetes Mellitus

DR - Diabetic Retinopathy

NPDR - Non Proliferative Diabetic Retinopathy

PDR- Proliferative Diabetic Retinopathy

CSME- Clinically Significant Macular Edema

ANOVA - Analysis Of Variance

Authors Competing Interests- 'The authors declare that they have no competing interests'

Financial or other competing interests: None, Acknowledgements: Nil

\section{Funding: Nil, Conflict of interest: None}

Permission of IRB: Yes

\section{References}

1. Rema M, Premkumar S, Anitha B, Deepa R, Pradeepa R, Mohan V. Prevalence of diabetic retinopathy in urban India: the Chennai Urban Rural Epidemiology Study (CURES) eye study, I. Invest Ophthalmol Vis Sci. 2005 Jul;46(7): 2328-33.

2. Morimoto Y, Conroy SM, Ollberding NJ, Kim Y, Lim U, Cooney RV, Franke AA, Wilkens LR, Hernandez BY, Goodman MT, Henderson BE, Kolonel LN, Le Marchand L, Maskarinec G. Ethnic differences in serum adipokine and C-reactive protein levels: the multiethnic cohort. Int J Obes (Lond). 2014 Nov;38(11):1416-22. doi: 10.1038 /ijo. 2014.25. Epub 2014 Feb 13.

3. Kilpatrick ES, Keevil BG, Jagger C, Spooner RJ, Small M. Determinants of raised C-reactive protein concentration in type 1 diabetes. QJM. 2000 Apr; 93 (4):231-6.

4. Early Treatment Diabetic Retinopathy Study design and baseline patient characteristics. ETDRS report number 7. Ophthalmology. 1991 May;98 (5 Suppl):741-56.
5. Schmidt AM, Hori O, Chen JX, Li JF, Crandall J, Zhang J, Cao R, Yan SD, Brett J, Stern D. Advanced glycation endproducts interacting with their endothelial receptor induce expression of vascular cell adhesion molecule-1 (VCAM-1) in cultured human endothelial cells and in mice. A potential mechanism for the accelerated vasculopathy of diabetes. J Clin Invest. 1995 Sep; 96 (3):1395-403.

6. Schram MT, Chaturvedi N, Schalkwijk C, Giorgino F, Ebeling P, Fuller JH, Stehouwer CD; EURODIAB Prospective Complications Study. Vascular risk factors and markers of endothelial function as determinants of inflammatory markers in type 1 diabetes: the EURODIAB Prospective Complications Study. Diabetes Care. 2003 Jul;26 (7):2165-73.

7. Yuuki T, Kanda T, Kimura Y, Kotajima N, Tamura J, Kobayashi I, Kishi S. Inflammatory cytokines in vitreous fluid and serum of patients with diabetic vitreoretinopathy. J Diabetes Complications. 2001 Sep-Oct;15(5):257-9.

8. Funatsu H, Yamashita H, Ikeda T, Mimura T, Shimizu E, Hori S. Relation of diabetic macular edema to cytokines and posterior vitreous detachment. Am J Ophthalmol. 2003 Mar;135 (3): 321-7.

9. Boyer DS, Yoon YH, Belfort R Jr, Bandello F, Maturi RK, Augustin AJ, Li XY, Cui H, Hashad Y, Whitcup SM; Ozurdex MEAD Study Group. Three-year, randomized, sham-controlled trial of dexamethasone intravitreal implant in patients with diabetic macular edema. Ophthalmology. 2014 Oct; 121(10):1904-14. doi: 10.1016/j.ophtha. 2014. 04. 024. Epub 2014 Jun 4.

10. Schalkwijk CG, Poland DC, van Dijk W, Kok A, Emeis JJ, Dräger AM, Doni A, van Hinsbergh VW, Stehouwer CD. Plasma concentration of Creactive protein is increased in type $\mathrm{I}$ diabetic patients without clinical macroangiopathy and correlates with markers of endothelial dysfunction: evidence for chronic inflammation. Diabetologia. 1999 Mar;42(3):351-7.

\section{How to cite this article?}

May D, Francis EA R, Norman M, Nelly N, Nameeth D, Shah A. J. Role of C- reactive protein in diabetic retinopathy. Int J Med Res Rev 2017;5(06):585-592. doi:10.17511/ijmrr. 2017.i06.07. 